\title{
Optimization of wide-band quasi-omnidirectional 1-D photonic
}

\section{structures}

\author{
V. Castillo-Gallardo ${ }^{a, b, c, *}$, Luis Eduardo Puente-Díaz ${ }^{a, b, c}$, D. Ariza-Flores ${ }^{d}$, \\ Héctor Pérez-Aguilar ${ }^{c}$, W. Luis Mochán ${ }^{b}$ and V Agarwal ${ }^{a, 1}$
}

\author{
${ }^{a}$ Centro de Investigación en Ingeniería y Ciencias Aplicadas, Universidad del Estado de Morelos, Av. Universidad 1001, Col. Chamilpa, \\ Cuernavaca, Morelos 62209, México. \\ ${ }^{b}$ Instituto de Ciencias Físicas, Universidad Nacional Autónoma de México, Av. Universidad S/N, Col. Chamilpa, 62210 Cuernavaca, Morelos, \\ México. \\ ${ }^{c}$ Facultad de Ciencias Físico Matemáticas, Universidad Michoacana de San Nicolás de Hidalgo, Av. Francisco J. Múgica S/N 58030, Morelia, \\ Mich., México. \\ ${ }^{d}$ CONACyT-Universidad Autónoma de San Luis Potosí, Karakorum 1470, Lomas 4ta Secc, San Luis Potosí, S.L.P., 78210, México.
}

\section{ARTICLE INFO}

\section{Keywords:}

Optimized reflectance

Omnidirectional structures

Chirped structures

Stacked Bragg mirror structures

\begin{abstract}
A B S T R A C T
We have designed, optimized, fabricated and characterized highly reflective quasi-omnidirectional (angular range of $0-60^{\circ}$ ) multilayered structures with a wide spectral range. Two techniques, chirping (a continuous change in thicknesses) and stacking of Bragg-type sub-structures, have been used to enhance the reflectance with minimum thickness for a given pair of refractive indices. Numerical calculations were carried out employing the transfer matrix method and we optimized the design parameters to obtain maximal reflectance averaged over different spectral ranges for all angles. We fabricated some of the optimized structures with porous silicon dielectric multilayers with low refractive contrast and compared their measured optical properties with the calculations. Two chirped structures with thicknesses $21.6 \mu \mathrm{m}$ and $60.4 \mu \mathrm{m}$, resulting in quasi omnidirectional mirrors with bandwidths of $360 \mathrm{~nm}$ and $1800 \mathrm{~nm}$, centered at $1160 \mathrm{~nm}$ and $1925 \mathrm{~nm}$ respectively have been shown. In addition, we fabricated a stacked sub-Bragg mirror structure with a quasi omnidirectional bandwidth of $1800 \mathrm{~nm}$ (centered at $1850 \mathrm{~nm}$ ) and a thickness of $41.5 \mu \mathrm{m}$, which is almost two third (in thickness) of the chirped structure. Thus, our techniques allowed us to obtain relatively thin quasi-omnidirectional mirrors with wide bands over different wavelength ranges. Our analysis techniques can be used for the optimization of the reflectance not only in multilayered PS systems with different refractive index contrasts but also for systems with other types of materials with low refractive index contrast. The present study could also be useful for obtaining omnidirectional dielectric mirrors in large spectral regions using different materials, flat focusing reflectors, thermal regulators, or, if defects are included, as filters or remote chemical/biosensors with a wide angular independent response.
\end{abstract}

\section{Introduction}

Photonic crystal (PC)-based structures have been extensively investigated due to their light controlling properties [1, 2]. Photons entering a PC interact with its periodically varying dielectric constant and their energies are consequently organized into photonic bands. In analogy to electronic bands withiny a crystal, propagation is forbidden if the energy lies within certain regions known as photonic band gaps (PBG's). Thus, the PC behaves as a mirror if illuminated by light with frequencies within in a PBG. For several applications it is useful to fabricate ommnidirectional mirrors with an absolute band gap for all angles of incidence. The use of metallic mirrors is limited due to their relatively large absorption at the visible (Vis) and near infrared (NIR) frequencies. An attractive alternative consists of low-absorption dielectric structures, which may be designed for specific frequency ranges. One-dimensional (1D) and two-dimensional (2D) PCs have applications in optoelectronics, optical telecommunications and computing, laser technology [3, 4], and radiative cooling applications [5]. The simplest 1D-PC is composed of a finite number of periodically alternating layers of high $n_{H}$ and low $n_{L}$ refractive indices, with corresponding thicknesses $d_{H}$ and $d_{L}$ chosen so that their optical

\footnotetext{
*Corresponding author

ing.victorcg@gmail.com (V. Castillo-Gallardo); fmatpuente@gmail.com (L.E. Puente-Díaz); david1cool@gmail.com (D. Ariza-Flores); hiperezag@yahoo.com (H. Pérez-Aguilar); mochan@fis.unam.mx (W.L. Mochán); vagarwal@uaem.mx (V. Agarwal) ORCID(s): 0000-0002-8157-3534 (V. Castillo-Gallardo); 0000-0003-3719-8535 (L.E. Puente-Díaz); 0000-0002-1775-6598 (D. Ariza-Flores); 0000-0002-8572-1485 (H. Pérez-Aguilar); 0000-0003-0418-5375 (W.L. Mochán); 0000-0003-2168-853X (V. Agarwal)

${ }^{1}$ On sabatical leave at ICF-UNAM from CIICAP-UAEM
} 
thicknesses corresponds to a quarter of a wavelength $n_{H} d_{H}=n_{L} d_{L}=\lambda_{0} / 4$ at a nominal wavelength $\lambda_{0}$. This yields a Bragg mirror (BM) which might have a large PBG including an omnidirectional gap if the contrast $n_{H} / n_{L}$ and the number of periods are large enough. Mirrors with high reflectance within a wider frequency range may be engineered by using different nominal wavelengths for different layers. One way to obtain such structures is to gradually change the width of the layers as a function of their depth, producing chirped-type structures [6]. Another alternative is to stack BMs, in such a way that their reflection bands partially overlap each other at their edges, resulting in a wider high-reflection band [7].

On the other hand, different fabrication techniques have been used to obtain omnidirectional mirrors (ODM) that operate on the visible and/or near infrared (Vis-NIR) range of the electromagnetic spectrum. For example, Chen et al. [8], reported the fabrication of a structure made of six pairs of $\mathrm{TiO}_{2} / \mathrm{SiO}_{2}$ layers with an omnidirectional band (ODB) of about $70 \mathrm{~nm}$ in the NIR range, employing a sol-gel deposition method. Park et al. [9], used molecular beam epitaxy to grow a stack of four pairs of GaAs/AlAs layers, followed by its conversion to $\mathrm{GaAs}_{\mathrm{Al}} \mathrm{Al}_{2} \mathrm{O}_{3}$ by selective oxidation of the AlAs layers, to obtain an ODB from 710 to $950 \mathrm{~nm}$. On the other hand, DeCorby et al. [10], fabricated mirrors by coupling multiple layers of $\mathrm{Ge}_{33} \mathrm{As}_{12} \mathrm{Se}_{55}$ chalcogenide glass and polyamide-imide deposited by thermal evaporation and spin-casting respectively, to obtain a $150 \mathrm{~nm}$ wide omnidirectional band centered at $1750 \mathrm{~nm}$. Furthermore, Jena et al. [11], used sequential asymmetric bipolar pulsed DC magnetron sputtering for $\mathrm{TiO}_{2}$ layers and radio frequency magnetron sputtering for $\mathrm{SiO}_{2}$ layers to generate $\mathrm{TiO}_{2} / \mathrm{SiO}_{2}$ 1D-PC's and achieved an ODB from 592 to $668 \mathrm{~nm}$. However, these techniques are expensive and they require sophisticated equipment and long fabrication times. Use of porous silicon (PS) is an attractive alternative, since it can be easily manufactured by electrochemical etching crystalline $\mathrm{Si}$ in an hydrofluoric acid based electrolyte to obtain a sponge-like nanostructure composed of Si and air. By modulating the supplied current and its application time during the electrochemical reaction, it is possible to obtain multilayered structures allowing for the fabrication ODM's [12, 13]. Although optical filters [14, 15] are the most common application of PS PC's, they have also been widely used as chemical sensors [16, 17], waveguides [18] and for photoluminescence control [19]. Recently, the study of chirped multilayer structures has increased due to their possible application as flat focusing mirrors [20,21, 22]. PS based dielectric optical filters, quasi-ODM's and ODM's have been extensively studied in different regions of the electromagnetic spectrum, such as the ultraviolet (UV) [23], visible [24], and NIR [25] regions. However, to obtain the desirable high index of refraction contrast, layers of high porosity have to be employed, which makes the resulting structures relatively fragile. In addition, use of high porosity makes the fabrication of large structures, with a very large number of layers, unfeasible. For this reason, in this work we study different strategies to produce multilayered mirrors with high reflectance omnidirectional band with relatively small thickness and refractive index contrast.

This paper is organized as follows. In Sec. 2 we develop the methods used to design and calculate the reflectance spectra of the PS multilayered structures such as chirped structures and mirror stacks. In Sec. 3 we provide details about the fabrication of these structures. In Sec. 4 we present the numerical and experimental results corresponding to our proposed structures and compare them to previous reports. We obtained significantly large band-widths with relatively thinner structures than reported previously. Finally, we discuss our conclusions in Section 5.

\section{Theory}

For the analysis of the propagation of electromagnetic fields through multilayered systems it is usual to employ the transfer matrix method [26, 27]. Assuming the system is $1 \mathrm{D}$, with variations only along the $z$ directions, and that the polarization of light is either transverse electric (TE) or transverse magnetic (TM), the transfer matrix $M\left(z_{2}, z_{1}\right)$ is a $2 \times 2$ matrix that relates the components of the electric $E_{\|}$and magnetic $H_{\|}$fields parallel to the $x y$ plane evaluated at $z_{2}$ to their value at $z_{1}$,

$$
\left(\begin{array}{c}
E_{\|} \\
H_{\|}
\end{array}\right)_{z_{2}}=M\left(z_{2}, z_{1}\right)\left(\begin{array}{c}
E_{\|} \\
H_{\|}
\end{array}\right)_{z_{1}} .
$$

Many equivalent formulations have been proposed to obtain and use $\boldsymbol{M}$ [28, 29, 30]. Here we use a recently developed formalism for the numerically stable calculation of the reflectance of large multilayer systems, as proposed by PuenteDíaz, et al. [31], summarized in the supplementary information. The explicit expressions for the optical coefficients are:

$$
r=\sigma \frac{Z_{0} M_{11}+M_{12}-Z_{0} Z_{s} M_{21}-Z_{s} M_{22}}{Z_{0} M_{11}-M_{12}-Z_{0} Z_{s} M_{21}+Z_{s} M_{22}},
$$


and

$$
t=\frac{2 \zeta}{Z_{0} M_{11}-M_{12}-Z_{0} Z_{s} M_{21}+Z_{s} M_{22}},
$$

where $M_{i j}$ are the elements of the transfer matrix which transfer the fields from its surface interface with vacuum at $z_{0}$ towards the substrate at $z_{s}, Z_{0}$ is the surface impedance of vacuum and $Z_{s}$ is the surface impedance of the substrate, and we define $r=F_{r} / F_{i}$ and $t=F_{t} / F_{i}$ in terms of the reflected and transmitted fields, where we choose the $F$ 's as electric fields for (TE) polarization and magnetic fields for transverse magnetic polarization (TM). Here, $\sigma=-1$ and $\zeta=Z_{s}$ for TE polarization, while $\sigma=1$ and $\zeta=Z_{0}$ for TM polarization. The reflectance is given by $R=|r|^{2}$ and the transmittance by $T=\beta|t|^{2}$ with $\beta=\operatorname{Re} Z_{0} / \operatorname{Re} Z_{s}$ for TE polarization and $\beta=\operatorname{Re} Z_{s} / \operatorname{Re} Z_{0}$ for TM polarization. The complex refractive index of the porous layers were obtained through the Bruggeman's effective medium theory, which has been reported to adequately reproduce the optical parameters of PS [16, 32, 33].

\section{Experimental details}

Some of the proposed photonic structures were synthesized through anodic etching of a (100) oriented, $p$-type Boron doped, crystalline Si wafer with resistivity 0.002-0.005 $\Omega \cdot \mathrm{cm}$, under galvanostatic conditions [34, 35]. The electrochemical anodization process was performed at room temperature, with an electrolyte of aqueous hydrofluoric acid (HF) (48\% of wt) and ethanol (99.9\% of wt) in 1:1 volumetric proportion, respectively. However, as it is not desirable to use very high porosity contrasts due to structure fragility and electrolyte diffusivity problems [36], the current densities were chosen as 35 and $305 \mathrm{~mA} / \mathrm{cm}^{2}$, with corresponding porosities of $51 \%$ and $76 \%$, respectively. These porosities were determined through previously obtained calibration curves using a gravimetric technique [32]. The etching rate of PS was obtained by synthetizing single layers under similar conditions and measuring their thicknesses through Scanning Electron Microscopy (SEM). Absolute reflectivity measurements were carried out with a Perkin Elmer Lambda $950 \mathrm{UV} /$ Visible spectrophotometer with a variable angle universal reflectance accessory (URA) for different incident angles $\theta_{i}=10^{\circ}, 20^{\circ}, 30^{\circ}, 40^{\circ}, 50^{\circ}$ and $60^{\circ}$ using non-polarized light. The maximum and minimum values of $\theta_{i}$ were constrained by the angular range of the URA.

\section{Results and discussion}

In this section we present optimized calculations of the reflectance of different multilayered omnidirectional wideband mirrors and we compare the results with experimental spectra taken from the corresponding samples at diferent angles of incidence. Sec. 4.1 is devoted to chirped-type Bragg mirrors while Sec. 4.2 is devoted to structures made of stacked sub-mirrors.

We develop two techniques to design multilayer photonic structures. In the first, the wavelength $\left(\lambda_{j}\right)$ to which the $j$-th pair of layers is tuned is determined by a given function of $j$. Several functions are proposed and their parameters are optimized to maximize the reflectance $\langle R\rangle$ averaged over a given spectral and angular range. The second technique consisted in stacking sub-mirrors tuned to different wavelengths, chosen so that their spectral ranges overlap. For each sub-mirror, the spectral range was obtained from the dispersion relation of a corresponding periodic structure. The degree of overlap was optimized to maximize $\langle R\rangle$. The optimizations were done employing the Minuit module [37] of the Perl Data Language (PDL) [38].

\subsection{Chirped-type Bragg mirrors}

Here we study multilayered structures where the thicknesses for each pair $j=0 \ldots N_{p}-1$ of layers are tuned to a wavelength $\lambda_{j}$ which changes gradually with the depth of the layer, according to

$$
\lambda_{j}=\lambda_{\min }+\left(\lambda_{\max }-\lambda_{\min }\right) f\left(\frac{j}{N_{p}-1}\right),
$$

where $\lambda_{\min }$ and $\lambda_{\max }$ are the minimum and maximum design wavelengths respectively, $N_{p}$ is the number of periods in the structure, and $f(\xi)$ is a smooth function that goes from 0 to 1 as its argument goes from the surface $(\xi=0)$ to the substrate $(\xi=1)$. We only consider increasing functions $f$ due to the high absorption of PS in the ultraviolet region, 
Optimization of wide-band quasi-omnidirectional 1-D photonic structures

\begin{tabular}{rrrrrrrrrr} 
Class & $\alpha$ & $\beta$ & $\mathrm{A}$ & $\lambda_{\min }(n m)$ & $N_{p}$ & $d(\mu \mathrm{m})$ & $\mathrm{ODW}(\mathrm{nm})$ & $\mathrm{ODC}(\mathrm{nm})$ & $\langle R\rangle(\%)$ \\
\hline \hline$f_{1}$ & 0.24 & - & - & 250 & 222 & 86.1 & 300 & 1150 & 89 \\
$f_{2}$ & 0.37 & 1.06 & - & 400 & 102 & 35.9 & 220 & 1110 & 88 \\
$f_{3}$ & $\mathbf{1 . 2 3}$ & $\mathbf{0 . 5 4 0}$ & $\mathbf{0 . 1 8}$ & $\mathbf{3 2 0}$ & $\mathbf{6 3}$ & $\mathbf{2 1 . 6}$ & $\mathbf{2 5 0}$ & $\mathbf{1 1 2 5}$ & $\mathbf{8 9}$ \\
\hline$f_{1}$ & $\mathbf{1 . 2 0}$ & - & - & - & $\mathbf{9 0}$ & $\mathbf{6 0 . 4}$ & $\mathbf{2 1 5 0}$ & $\mathbf{1 9 2 5}$ & $\mathbf{9 2}$ \\
$f_{2}$ & 1.08 & 1.86 & - & - & 105 & 61.7 & 2000 & 2000 & 89 \\
$f_{3}$ & 1.01 & 1.95 & 0.20 & - & 102 & 62.3 & 2050 & 1975 & 90
\end{tabular}

Table 1

Optimized parameters $\alpha, \beta, A$ and $N_{p}$ yielding the highest reflectance $\langle R\rangle$ averaged over the spectral ranges $\mathcal{R}_{1}$ (first block) and $\mathcal{R}_{2}$ (second block) and the angular range $0-90^{\circ}$ for the profile classes $f_{1}, f_{2}$ and $f_{3}$ (Eqs. (4) - (6)). We include the thickness $d$ of the structure and the omnidirectional spectral width and center (ODW and ODC).

which decreases in the visible and becomes negligible in the near infrared, so, that the first periods are tuned in the $\mathrm{UV}-\mathrm{Vis}$ regions. We consider the following classes of functions:

$$
\begin{aligned}
& f_{1}(\xi)=\xi^{\alpha}, \\
& f_{2}(\xi)=\frac{1}{2}\left(\xi^{\alpha}+\xi^{\beta}\right)
\end{aligned}
$$

and

$$
f_{3}(\xi)=A \xi^{\alpha}(1-\xi)+\xi^{\beta},
$$

where $\alpha, \beta$ and $A$ are parameters to optimize in order to maximize the average reflectance $\langle R\rangle$ over given spectral and angular ranges. The class $f_{1}$ (Eq. (4)) with $\alpha>0$ corresponds to simple increasing profiles, $f_{2}$ is the arithmetic mean of two functions of the type $f_{1}$ with different powers $\alpha$ and $\beta$, and $f_{3}$ (Eq. (6)) is designed so that the first term dominates near the surface and the second near the substrate, to allow different behaviors at the edges of the spectrum. The parameters $\alpha, \beta, A$ and $N_{p}$ were optimized to maximize the reflectance for non-polarized light $\langle R\rangle$ averaged in the angular range of $0^{\circ}$ to $90^{\circ}$ and either in the spectral region $\mathcal{R}_{1}$ from 350 to $1400 \mathrm{~nm}$ or $\mathcal{R}_{2}$ from 400 to $3000 \mathrm{~nm}$. For $\mathcal{R}_{1}$ we took $\lambda_{\min }$ as another parameter to be optimized while $\lambda_{\max }$ was fixed at $1400 \mathrm{~nm}$. For $\mathcal{R}_{2}$ we fixed both $\lambda_{\text {min }}=850 \mathrm{~nm}$ and $\lambda_{\text {min }}=3000 \mathrm{~nm}$. In the first case, we decided to optimize $\lambda_{\text {min }}$ to obtain the best design in the high absorption zone of the PS. Approximately $90 \%$ of the solar radiation that reaches the earth's surface is contained in the spectral region $\mathcal{R}_{1}$, and $98 \%$ in $\mathcal{R}_{2}$ [39]. A direct application of the optimized structures in these regions is solar reflectors. The optimized parameters corresponding to the choice $p_{l}=0.51$ and $p_{h}=0.76$ for the low and high porosities are shown in Table 4.1. In the supplementary information, we include the parameters used to calculate the reflectance of structures corresponding to the porosity $p_{l}=0.30$ and $p_{h}=0.76$. We also add the theoretical results obtained for $p_{l}=0.42$ and $p_{h}=0.76$.

The function $f_{3}$ yields after optimization the highest $\langle R\rangle$ in the region $\mathcal{R}_{1}$ and with the thinnest structure. Furthermore, this structure has an omnidirectional width (ODW) of $250 \mathrm{~nm}$ centered at (ODC) $1125 \mathrm{~nm}$, as shown in Fig. 4.1(b). Various photonic structures have been reported with ODB's [40, 41, 42] defined as those spectral regions for which $R>90 \%$ for all angles. Here we we adopt the same criterium. We synthesized a structure designated sample $S_{1}$ corresponding to these results. For the region $\mathcal{R}_{2}$ the resulting structures have similar thicknesses and attain similar average reflectance, though the one with the smallest thickness and the maximum $\langle R\rangle$ is that obtained from the family $f_{1}$. We also synthesized the corresponding structure and designated it sample $S_{2}$. The calculated optimized reflectance spectrum is shown in Fig. 4.1(d). This structure has a very wide region where $R(\lambda, \theta)>0.90$, which goes from 850 to $3000 \mathrm{~nm}$, but in a restricted angular range from $0^{\circ}$ to $70^{\circ}$. Thus, it has a quasi-ODW of $2150 \mathrm{~nm}$ centered at $1925 \mathrm{~nm}$. The functions $f_{n}$ optimized for the region $\mathcal{R}_{1}$ are shown in Fig. 4.1(a), and those for $\mathcal{R}_{2}$ in Fig. 4.1(c). The profiles $f_{n}$ are qualitatively similar. For example, for $\mathcal{R}_{1}$ the optimal photonic structures have a small number of periods tuned to the wavelengths where silicon has a large absorption, i.e., the first periods of the structure. As withinin $\mathcal{R}_{2}$ the absorption of porous silicon is negligible, the optimized $f_{n}$ are not too far from a linear profile.

In Fig. 4.1(a) we present the calculated and measured reflectance for non-polarized light corresponding to sample $S_{1}$ in the spectral range from 350 to $1400 \mathrm{~nm}$ for different angles of incidence $\theta=10^{\circ}, 20^{\circ}, 30^{\circ}, 40^{\circ}, 50^{\circ}$, and $60^{\circ}$. The 

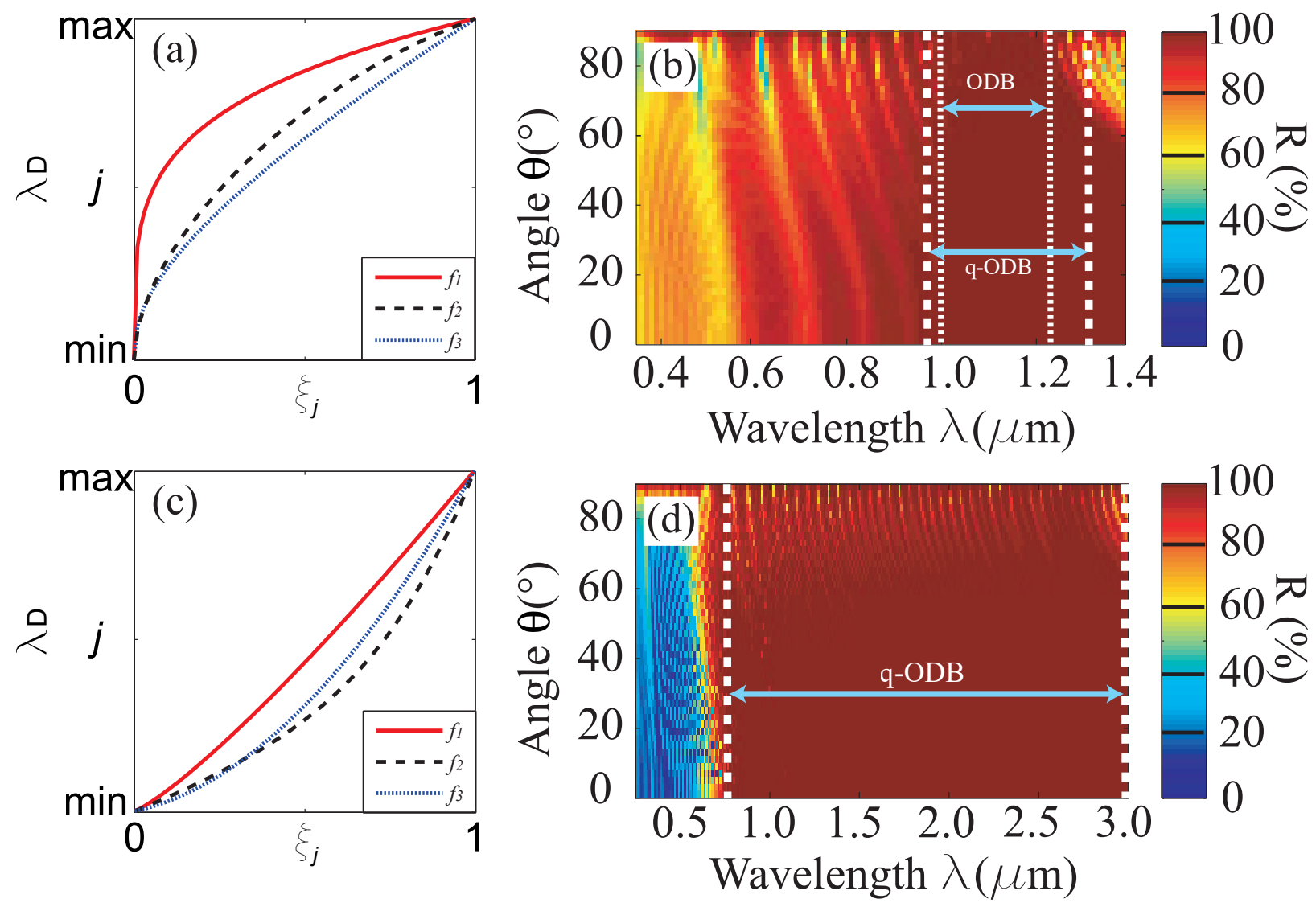

Figure 1: Profiles of the functions $f_{n}\left(\xi_{j}\right)$ optimized for the region $\mathcal{R}_{1}$ from 350 to $1400 \mathrm{~nm}$ (panel a) and $\mathcal{R}_{2}$ from 400 to $3000 \mathrm{~nm}$ (panel c). Reflectance for non-polarized light vs. wavelength and angle of incidence of the structure optimized for the region $\mathcal{R}_{1}$ (panel b) and $\mathcal{R}_{2}$ (panel d). The dotted lines indicate the ODB and the dashed lines indicate the quasi-ODB.

spectra are qualitatively similar, and for $\lambda>700 \mathrm{~nm}$ and $10^{\circ}<\theta<40^{\circ}$, they agree quantitatively, with a difference smaller than $5 \%$. The measured reflectance is lower than the calculated one, more so at small wavelengths and large angles. This difference may be partially attributed to the scattering of light due to the roughness present at the sample $[43,30,29]$ that we have not taken into account in our theory. Due to the limitations of our spectrophotometer, we couldn't perform measurements for $\theta>60^{\circ}$ and thus check the ODB. Nevertheless, our results allow us to conclude that the structure has a quasi-ODB from 980 and $1340 \mathrm{~nm}$. In Fig. 4.1(b) we compare the calculated and measured spectra of sample $S_{2}$ in the range from 400 to $3000 \mathrm{~nm}$. Both spectra are very similar, with some differences at long wavelengths which can be attributed to an increase of imperfections such as wavy layers and more roughness for deeper layers, due to a more restricted diffusivity of the electrolyte as the thickness of the structure is increased $[44,45]$. According to our results, this structure has a wide quasi-ODW of $1800 \mathrm{~nm}$ centered at $1880 \mathrm{~nm}$.

\subsection{Photonic structure with sub-mirrors stacking}

Another class of techniques for designing good reflectors across a wide spectral range is to stack sub-mirrors tuned to different wavelengths chosen with different criteria. For example, Agarwal et al. [46] proposed a photonic structure in which 54 sub-mirrors were stacked as follows: the first sub-mirror consisted of 2.5 periods tuned to $\lambda_{1}=700 \mathrm{~nm}$; the following $j$-th sub-mirrors were tuned to wavelengths $\lambda_{j}$ according to the sequence $\lambda_{j+1}=\lambda_{j}+(2+j) \mathrm{nm}$ with 4 periods for each of the following periods. Furthermore, Estrada-Wiese et al. [47] developed a method that combines three stochastic optimization algorithms together with a narrow space search methodology to obtain a customized and optimized PC configuration. 
(a)

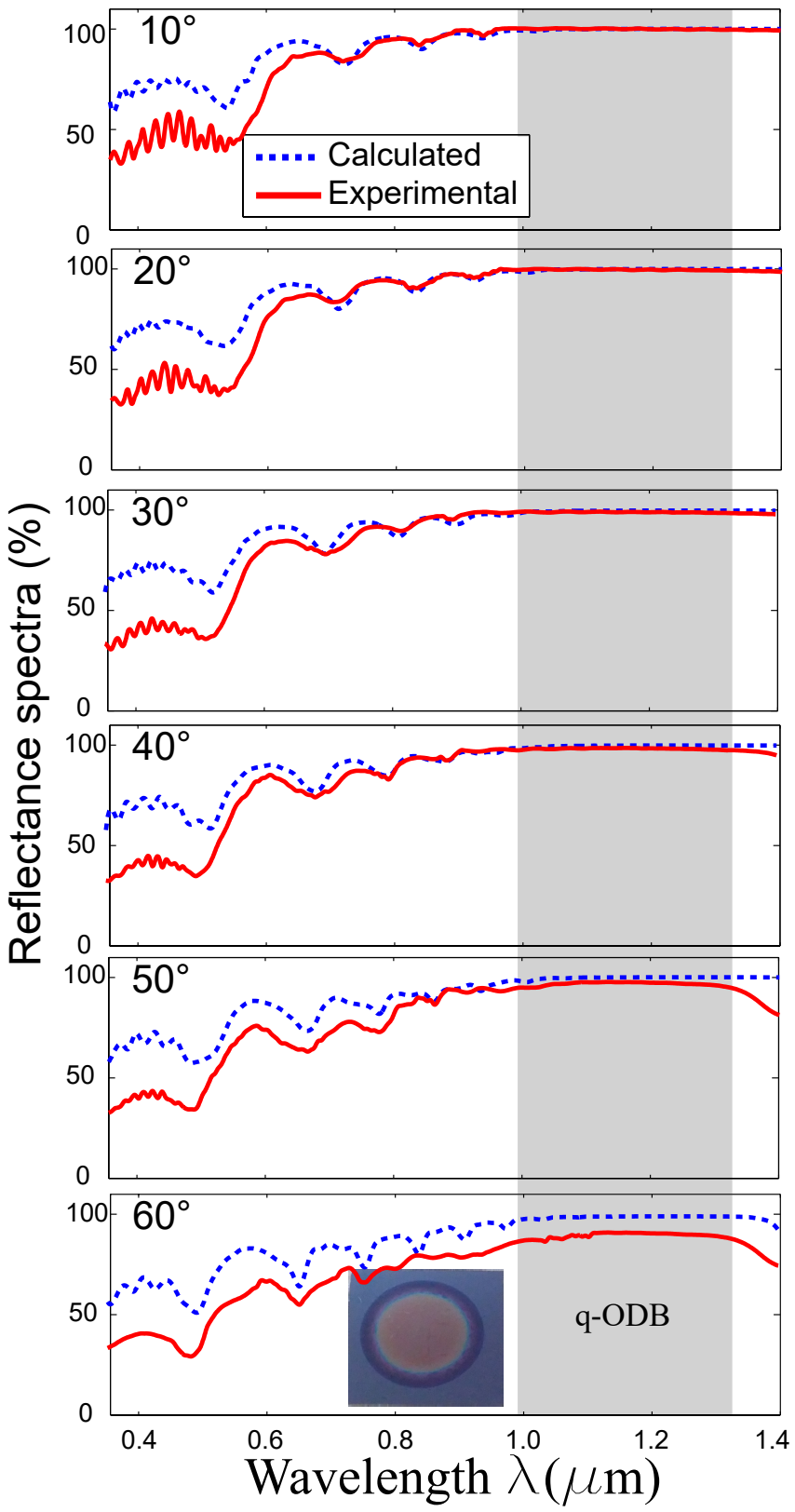

(b)
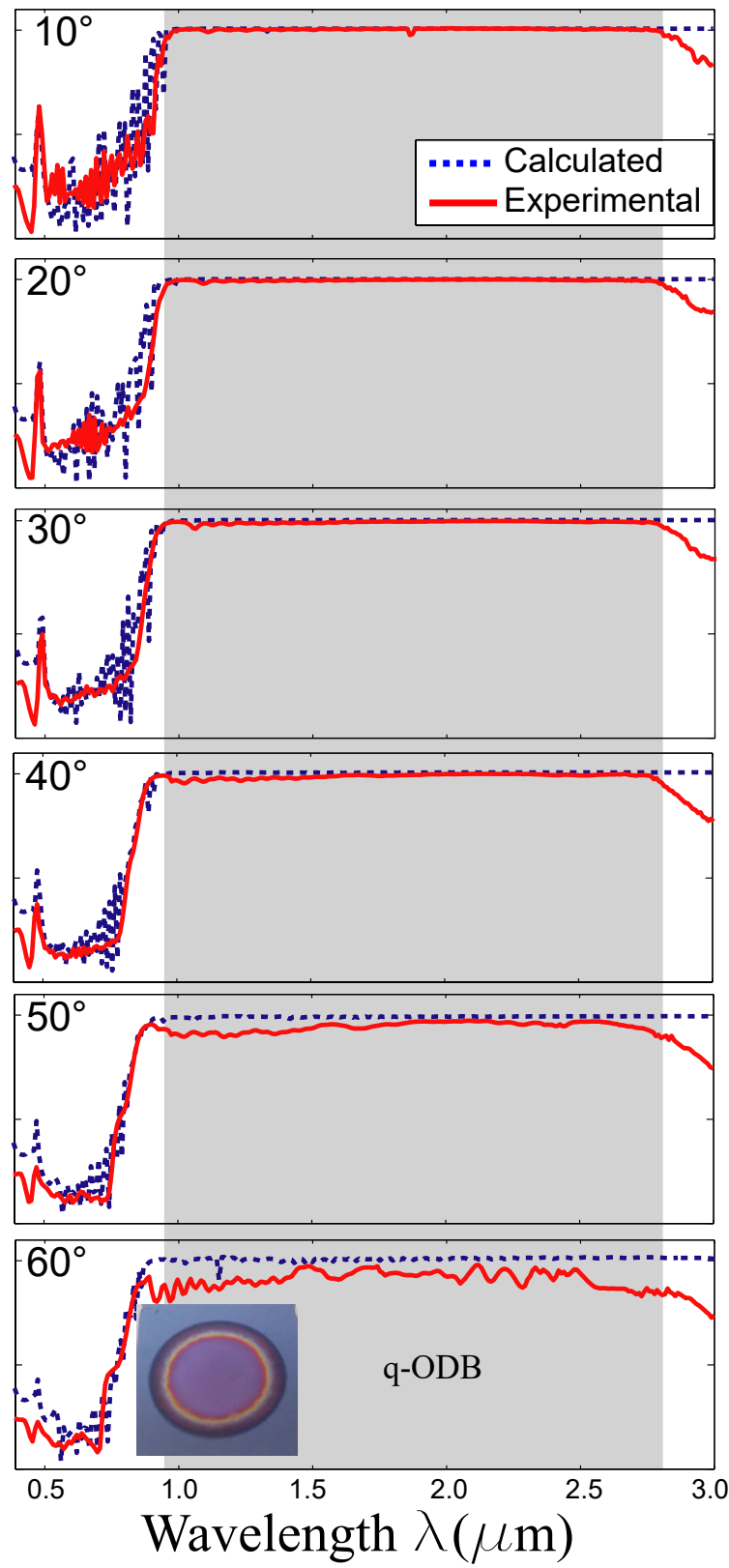

Figure 2: Reflectance spectra for non-polarized light calculated and measured at different angles of incidence $\theta=10^{\circ}$, $20^{\circ}, 30^{\circ}, 40^{\circ}, 50^{\circ}$, and $60^{\circ}$ for structure $S_{1}$ (panel a) and $S_{2}$ (panel b). The gray band indicates the region where the reflectance is greater than 0.90 , corresponding to the quasi ODB of the structure. Inset: photograph of the synthesized structure.

We recall that for a periodic structure, the propagation of electromagnetic waves is described by the dispersion relation

$$
\cos K D=\frac{1}{2} \operatorname{Tr} M
$$

where $K$ is Bloch's vector and $\boldsymbol{M}$ the transfer matrix of a single period of thickness $D[28,48,49]$. Thus, whenever 
Optimization of wide-band quasi-omnidirectional 1-D photonic structures

\begin{tabular}{rrrrr}
$p$ & $N_{m}$ & $d(\mu \mathrm{m})$ & \% overlap & $\langle R\rangle$ \\
\hline \hline 1 & 98 & 43.2 & 95 & 91.0 \\
3 & 31 & 42.5 & 81 & 91.8 \\
5 & 17 & 41.9 & 68 & 92.0 \\
\hline 4 & 25 & 38.7 & 74 & 92.3 \\
$\mathbf{6}$ & $\mathbf{1 8}$ & $\mathbf{4 1 . 5}$ & $\mathbf{7 8}$ & $\mathbf{9 5 . 4}$ \\
10 & 13 & 45.4 & 56 & 95.3
\end{tabular}

\section{Table 2}

Total number $N_{m}$ of stacked sub-mirrors, thickness $d$, overlap between consecutive PBG and average reflectance $\langle R\rangle$ for optimized structures designed with a number $p$ of periods per sub-mirror (first block) or a number $p$ of periods for those sub-mirrors tuned to wavelengths $\lambda_{j} \geq 800 \mathrm{~nm}$ (second block, see text), for different values of $p$. The parameters corresponding to the best design are typeset in bold.

$|\operatorname{Tr}(M)|>2$ electromagnetic energy cannot propagate in the periodic system. This condition corresponds to a photonic band gap within which we expect a high reflectance, even for a finite system made of only a few periods. This suggests the following design strategy. We stack sub-mirrors each made of a few periods of a periodic system such that its photonic band gap (PBG) overlaps the PBG of the previous and that of the next sub-mirror. The number of periods of each sub-mirror is an important parameter. If it is too small, the sub-mirror would be partially transparent within the PBG. If it is too large, the resulting structure would be too thick. This is particularly important for sub-mirrors tuned to shorter wavelengths, for which there is some absorption. We followed thus two design strategies: In the first, we considered sub-mirrors each with the same number $p$ of periods. In the second, a different number of periods was assigned to each sub-mirror according to the center of its PBG: we chose a single period for those sub-mirrors $j$ tuned to $\lambda_{j}<500 \mathrm{~nm}$, two periods for $500<\lambda_{j}<650 \mathrm{~nm}$, three periods for $650<\lambda_{j}<800 \mathrm{~nm}$, and for $\lambda_{j} \geq 800 \mathrm{~nm}$ the number of periods $p$ was varied between 4 and 10. In both designs, the degree of overlap of consecutive PBGs was taken as the parameter to be optimized in order to maximize the reflectance $\langle R\rangle$ averaged within the spectral and angular range $400 \mathrm{~nm} \leq \lambda \leq 3000 \mathrm{~nm}$ and for $0^{\circ} \leq \theta \leq 90^{\circ}$. We define the percentage of overlap in terms of the portion of the PBG of the $j$-th mirror that lies within the PBG of the $(j+1)$-th mirror.

Table 4.2 shows the optimized values of the average reflectance $\langle R\rangle$ for different choices of the number of periods $p$ of each sub-mirror, as discussed above, including the resulting number $N_{m}$ of stacked sub mirrors, the thickness $d$ of the structure, and the optimal overlap of consecutive PBGs. The first and second blocks correspond to the first and second strategies discussed above.

According to table 4.2, the structure that has the highest reflectance is in the second block, with an optimal overlap between consecutive PBGs of 78\%, a thickness of $41.5 \mu \mathrm{m}$, and an average reflectance $\langle 0.954\rangle$. It is made up of 18 submirrors tuned to the wavelengths 400, 460, 510, 570, 640, 720, 810, 910, 1020, 1150, 1290, 1450, 1630, 1830, 2060, 2320, 2610, and $2860 \mathrm{~nm}$. We synthesized this structure and designated it sample $S_{3}$. Its calculated and measured reflectance spectra are shown in Fig. 3.

Fig. 3(a) reveals a quasi-ODM in the range from 950 to $2900 \mathrm{~nm}$, with an angular range between $0^{\circ}$ and $60^{\circ}$. It is also observed that at incidence angles $\theta>70^{\circ}$ and long wavelengths the reflectance decreases and has oscillations, which we attribute to the greater penetration of the electromagnetic field within the structure, as shown by Puente-Díaz, et al. [31]. In Fig. 3(b) we compare the calculated and measured reflectance for the structure $S_{3}$ at several angles of incidence. Although, the quasi omnidirectional wavelength range of the synthesized structure, from 950 to $2750 \mathrm{~nm}$, is slightly smaller than the calculated one, the measured PBG is almost twice as wide as a recently reported PS chirped structure [30], which has the largest previously reported ODW.

Finally, Table 4.2 shows data from some porous silicon based ODM designed to operate in the NIR region and their of the ODB's are compared with those of the structures analyzed in the present study. The ODW of the structure $S_{1}$ results $20 \mathrm{~nm}$ wider than that reported by Bruyant, et al. [25] and it is located closer to the visible range. Furthermore, the ODB of the structure $S_{3}$ begins at $950 \mathrm{~nm}$ like the one synthesized by Estevez, et al. [14]; nevertheless, its width extends to $2750 \mathrm{~nm}$. In other words, $S_{3}$ has an ODW 3.55 times higher. Moreover, the ODW of the structure reported by Xifre-Perez, et al. [50] represents only $17.6 \%$ of the ODW of our $S_{2}$ and $S_{3}$ structures. Aditionally, the table reveals that the porous silicon based structures $S_{2}$ and $S_{3}$ have the widest gap widths, 1.36 times greater than the largest reported work until now in the NIR region [20]. As compared to the previous studies [25, 14, 50, 20, 51], this work opens the possibility of generating dielectric multilayered structure with large omnidirectional band with the 
(b)
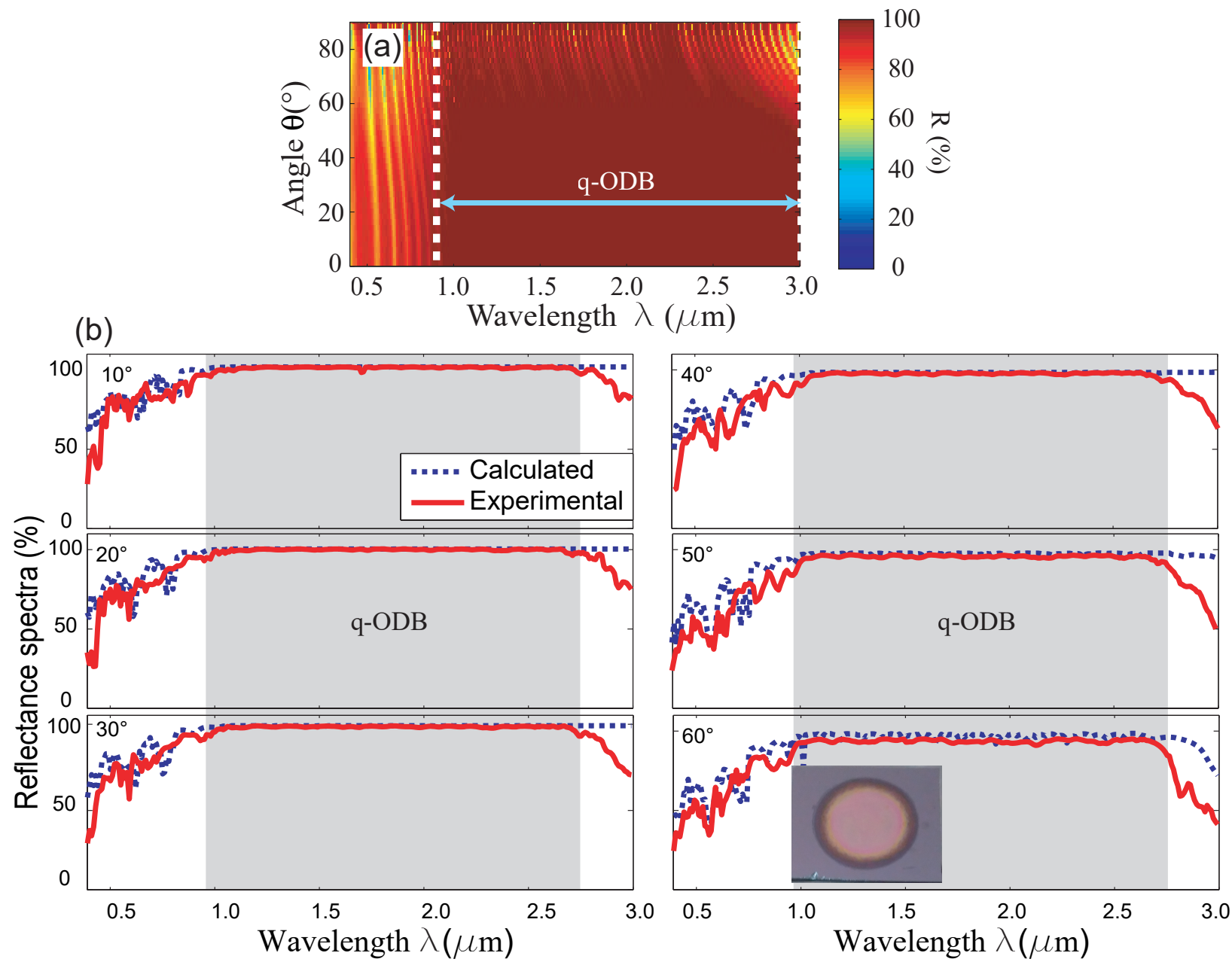

Figure 3: (a) Calculated reflectance $R(\lambda, \theta)$ for non-polarized light of the optimized structure $S_{3}$ as a function of wavelength $\lambda$ and incidence angle $\theta$. (b) Measured and calculated reflectance spectra at several angles of incidence $\theta=10^{\circ}, 20^{\circ}$, $30^{\circ}, 40^{\circ}, 50^{\circ}, 60^{\circ}$. The gray band indicates the region in which the reflectance is greater than $R>0.90$. Inset shows a photograph with a top view of the synthesized structure.

materials with relatively low refractive index contrast.

\section{Conclusion}

We have designed, optimized, fabricated and characterized highly reflective quasi omnidirectional (angular range of $0-60^{\circ}$ ) multilayered structures with a wide spectral range. Two techniques, chirping (a continuous change in thicknesses) and stacking of Bragg-type sub-structures, have been used to enhance the reflectance with minimum thickness for a given pair of refractive indices. Numerical calculations were carried out employing the transfer matrix method and we optimized the design parameters to obtain maximal reflectance averaged over different spectral ranges for all angles. We fabricated some of the optimized structures with porous silicon dielectric multilayers with low refractive contrast and compared their measured optical properties with the calculations. Two chirped structures with thicknesses $21.6 \mu \mathrm{m}$ and $60.4 \mu \mathrm{m}$, resulting in quasi omnidirectional mirrors with bandwidths of $360 \mathrm{~nm}$ and 1800 $\mathrm{nm}$, centered at $1160 \mathrm{~nm}$ and $1925 \mathrm{~nm}$ respectively have been shown. In addition, we fabricated a stacked sub-Bragg mirror structure with a quasi omnidirectional bandwidth of $1800 \mathrm{~nm}$ (centered at $1850 \mathrm{~nm}$ ) and a thickness of $41.5 \mu \mathrm{m}$, which is almost two third (in thickness) of the chirped structure. Thus, our techniques allowed us to obtain relatively 
Optimization of wide-band quasi-omnidirectional 1-D photonic structures

\begin{tabular}{rrrrr} 
Reference & Year & Sample & ODB $(\mathrm{nm})$ & ODW $(\mathrm{nm})$ \\
\hline \hline Bruyant, et al. [25] & 2003 & - & $1100-1440$ & 340 \\
Xifre-Pérez, et al. [50] & 2004 & - & $1297-1615$ & 318 \\
Estevez, et al. [14] & 2009 & - & $950-1456$ & 506 \\
Chavez, et al. [30] & 2020 & - & $1000-2000$ & 1000 \\
Wu, et al. [20] & 2021 & - & $1284-2605$ & 1321 \\
\hline & & $S_{1}$ & $980-1340$ & 360 \\
This work & 2021 & $S_{2}$ & $980-2780$ & 1800 \\
& & $S_{3}$ & $950-2750$ & 1800
\end{tabular}

\section{Table 3}

Development of porous silicon based omnidirectional mirrors for the NIR region for previously published works (first block) and for the different samples studied here (second block. We show the attained omnidirectional bands (ODB) and their widths (ODW).

thin quasi-omnidirectional mirrors with wide bands over different wavelength ranges. Our analysis techniques can be used for the optimization of the reflectance not only in multilayered PS systems with different refractive index contrasts but also for systems with other types of materials with low refractive index contrast. The present study could also be useful for obtaining omnidirectional dielectric mirrors in large spectral regions using different materials, flat focusing reflectors, thermal regulators, or, if defects are included, as filters or remote chemical/biosensors with a wide angular independent response.

\section{Acknowledgments}

This project is supported by Consejo Nacional de Ciencia y Tecnología through grant No.A-S1-30393(VA), 256243 (DA), Cátedras Conacyt program 1577 (DA), and by DGAPA-PAPIIT UNAM under grant IN111119 (LM). VA acknowledges PRODEP (Sabbatical grant), and is grateful to ICF where she spent the sabbatical year. HPA also expresses his gratitude to the Coordinación de la Investigación Científica de la Universidad Michoacana de San Nicolás de Hidalgo. VCG is grateful to CIICAp and ICF where he did this work.

\section{CRediT authorship contribution statement}

V. Castillo-Gallardo: Conceptualization, Software \& Investigation, synthesis \& characterization, Writing. Luis Eduardo Puente-Díaz: Conceptualization, Software \& Investigation. D. Ariza-Flores: Investigation, Writing - review \& editing. Héctor Pérez-Aguilar: Investigation and Supervision. W. Luis Mochán: Conceptualization, Funding acquisition, Investigation, Supervision, Writing - review \& editing. V Agarwal: Conceptualization, Funding acquisition, Investigation, Methodology, Supervision, Writing - review \& editing.

\section{References}

[1] John D. Joannopoulos, Steven G. Johnson, Joshua N. Winn, and Robert D. Meade. Optical Waves in Layered Media Photonic Crystals: Molding the Flow of Light. Princeton University Press, New Jersey, 2nd edition, 2008.

[2] Amit Kumar Goyal, Hemant Sankar Dutta, and Suchandan Pal. Porous photonic crystal structure for sensing applications. J. Nanophotonics, 12(4):1-7, 2018.

[3] C. López. Materials Aspects of Photonic Crystals. Adv. Mat., 15(20):1679-1704, 2003.

[4] Masaya Notomi. Manipulating light with strongly modulated photonic crystals. Rep. Prog. Phys., 73(9):096501, 2010.

[5] Amit Kumar Goyal and Ajay Kumar. Recent advances and progresses in photonic devices for passive radiative cooling application: A review. J. Nanophotonic, 14(3): 1-20, 2020.

[6] R. Szipöcs and A. Koházi-Kis. Theory and design of chirped dielectric laser mirrors. Appl. Phys. B, 65(2):115-135, 1997.

[7] E. Xifré-Pérez, L. F. Marsal, J. Ferré-Borrull, and J. Pallarés. Low refractive index contrast porous silicon omnidirectional reflectors. Appl. Phys. B, 95(1):169-172, 2009.

[8] Kevin M. Chen, Andrew W. Sparks, Hsin-Chiao Luan, Desmond R. Lim, Kazumi Wada, and Lionel C. Kimerling. $\mathrm{SiO}_{2} / \mathrm{TiO}_{2}$ omnidirectional reflector and microcavity resonator via the sol-gel method. Appl. Phys. Lett., 75(24):3805-3807, 1999.

[9] Yeonsang Park, Young-Geun Roh, Chi-O Cho, Heonsu Jeon, Min Gyu Sung, and J. C. Woo. GaAs-based near-infrared omnidirectional reflector. Appl. Phys. Lett., 82(17):2770-2772, 2003.

[10] R. G. DeCorby, H. T. Nguyen, P. K. Dwivedi, and T. J. Clement. Planar omnidirectional reflectors in chalcogenide glass and polymer. Opt. Express, 13(16):6228-6233, 2005. 
[11] S. Jena, R. B. Tokas, S. Tripathi, K. D. Rao, D. V. Udupa, S. Thakur, and N. K. Sahoo. Influence of oxygen partial pressure on microstructure, optical properties, residual stress and laser induced damage threshold of amorphous $\mathrm{HfO}_{2}$ thin films. J. Alloy Compd., 771:373-381, 2019.

[12] Elisabet Xifré-Pérez, Josep Ferré-Borrull, Josep Pallarés, and Lluís F. Marsal. Methods, Properties and Applications of Porous Silicon. In Dusan Losic and Abel Santos, editors, Electrochemically Engineered Nanoporous Materials: Methods, Properties and Applications, Springer Series in Materials Science, pages 37-63. Springer International Publishing, Cham, 2015.

[13] O. Bisi, Stefano Ossicini, and L. Pavesi. Porous silicon: a quantum sponge structure for silicon based optoelectronics. Surf. Sci. Rep., 38(1):1 $-126,2000$.

[14] J. O. Estevez, J. Arriaga, A. Méndez Blas, and V. Agarwal. Enlargement of omnidirectional photonic bandgap in porous silicon dielectric mirrors with a gaussian profile refractive index. Appl. Phys. Lett., 94(6):061914, 2009.

[15] D. Ariza-Flores, J.S. Pérez-Huerta, Yogesh Kumar, A. Encinas, and V. Agarwal. Design and optimization of antireflecting coatings from nanostructured porous silicon dielectric multilayers. Sol. Energ. Mat. Sol. C., 123:144-149, 2014.

[16] Anne M. Ruminski, Giuseppe Barillaro, Charles Chaffin, and Michael J. Sailor. Internally referenced remote sensors for $\mathrm{HF}$ and $\mathrm{Cl}_{2}$ using reactive porous silicon photonic crystals. Adv. Funct. Mat., 21(8):1511-1525, 2011.

[17] T.V.K. Karthik, L. Martinez, and V. Agarwal. Porous silicon $\mathrm{ZnO} / \mathrm{SnO}_{2}$ structures for $\mathrm{CO}_{2}$ detection. J. Alloy Compd., $731: 853$ - 863, 2018 .

[18] C. P. Hussell and R. V. Ramaswamy. High-index overlay for high reflectance DBR gratings in $\mathrm{LiNbO}_{3}$ channel waveguides. IEEE Photonic Tech. L., 9(5):636-638, 1997.

[19] E.E. Antunez, J.O. Estevez, J. Campos, M.A. Basurto-Pensado, and V. Agarwal. Formation of photoluminescent n-type macroporous silicon: Effect of magnetic field and lateral electric potential. Physica B, 453:34 - 39, 2014. Low-Dimensional Semiconductor Structures - A part of the XXII International Material Research Congress (IMRC 2013).

[20] F. Wu, K. Lyu, S. Hu, M. Yao, and S. Xiao. Ultra-large omnidirectional photonic band gaps in one-dimensional ternary photonic crystals composed of plasma, dielectric and hyperbolic metamaterial. Opt. Mater., 111:110680, 2021.

[21] Volokh Anatoliy, Sergey Marchenko, and Pavel Shestakov. Focusing and defocusing of reflected light beams from chirped dielectric layered structure. In 2017 Days on Diffraction (DD), pages 200-204, 062017.

[22] Yu-Chieh Cheng and Kestutis Staliunas. Near-field flat focusing mirrors. Appl. Phys. Rev., 5(1):011101, 2018.

[23] María Jimenez Vivanco, Godofredo García, Jesús Carrillo, Vivechana Agarwal, Tomás Díaz-Becerril, Rafael Doti, Jocelyn Faubert, and Eduardo Lugo. Porous $\mathrm{Si}_{-} \mathrm{SiO}_{2}$ based UV Microcavities. Sci. Rep.-UK, 10:2220, 122020.

[24] A. David Ariza-Flores, L. M. Gaggero-Sager, and V. Agarwal. White metal-like omnidirectional mirror from porous silicon dielectric multilayers. Appl. Phys. Lett., 101(3):031119, 2012.

[25] A. Bruyant, G. Lérondel, P. J. Reece, and M. Gal. All-silicon omnidirectional mirrors based on one-dimensional photonic crystals. Appl. Phys. Lett., 82(19):3227-3229, 2003.

[26] Rolando Perez-Alvarez and F. García-Moliner. Transfer matrix, Green Function and related techniques: Tools for the study of multilayer heterostructures. Universitat Jaume I, España, 012004.

[27] Pochi Yeh. Optical Waves in Layered Media. Wiley, USA, 2nd edition, 2005.

[28] Mochán W. Luis., del Castillo-Mussot Marcelo., and Barrera Rubén G. Effect of plasma waves on the optical properties of metal-insulator superlattices. Phys. Rev. B, 35(3):1088-1098, 1987.

[29] Leandro L. Missoni, Guillermo P. Ortiz, María Luz Martínez Ricci, Victor J. Toranzos, and W. Luis Mochán. Rough 1D photonic crystals: A transfer matrix approach. Opt. Mater., 109:110012, 2020.

[30] B. A. Chavez-Castillo, J. S. Pérez-Huerta, J. Madrigal-Melchor, S. Amador-Alvarado, I. A. Sustaita-Torres, V. Agarwal, and D. Ariza-Flores. A wide band porous silicon omnidirectional mirror for the near infrared range. J. Appl. Phys., 127(20):203106, 2020.

[31] Luis Eduardo Puente-Díaz, Victor Castillo-Gallardo, Guillermo P. Ortiz, José Samuel Pérez-Huerta, Héctor Pérez-Aguilar, Vivechana Agarwal, and W. Luis Mochán. Stable calculation of optical properties of large non-periodic dissipative multilayered systems. Superlattice Microst., 145:106629, 2020.

[32] Andrea Edit Pap, Krisztián Kordás, Jouko Vähäkangas, Antti Uusimäki, Seppo Leppävuori, Laurent Pilon, and Sándor Szatmári. Optical properties of porous silicon. Part III: Comparison of experimental and theoretical results. Opt. Mater., 28(5):506-513, 2006.

[33] D. Estrada-Wiese and J.A. del RÃo. Refractive index evaluation of porous silicon using bragg reflectors. Rev. Mex. Fís., 64:72-81, 02 2018.

[34] L. T. Canham. Silicon quantum wire array fabrication by electrochemical and chemical dissolution of wafers. Appl. Phys. Lett., 57(10):10461048, 1990. Publisher: American Institute of Physics.

[35] Escorcia J. and Agarwal V. Effect of duty cycle and frequency on the morphology of porous silicon formed by alternating square pulse anodic etching. Phys. Stat. Sol., 4(6):2039-2043, 2007.

[36] Ariza-Flores A. David, Gaggero-Sager L. M., and Agarwal V. Effect of interface gradient on the optical properties of multilayered porous silicon photonic structures. J. Phys. D: Appl. Phys., 44(15):155102, 2011.

[37] F. James. MINUIT Function Minimization and Error Analysis: Reference Manual Version 94.1. CERN Program Library Long Writeup D506, 1994.

[38] K. Glazebrook, J. Brinchmann, C. DeForest J. Cerney, D. Hunt, T. Jenness, T. Lukka, R. Schwebel, and C. Soeller. Perl Data Language (PDL), 1997.

[39] R.E. Bird, R.L. Hulstrom, and L.J. Lewis. Terrestrial solar spectral data sets. Solar Energy, 30(6):563-573, 1983.

[40] J. Xu, Q. Sun, Z. Wu, L. Guo, S. Xie, Q. Huang, and Q. Peng. Development of broad-band high-reflectivity multilayer film for positron emission tomography system. Journal of Instrumentation, 13(09):P09016-P09016, 2018.

[41] Kamal Ahmed, A Khan, A Rauf, and Amir Gul. Multilayer dielectric narrow band mangin mirror. IOP Conference Series: Materials Science and Engineering, 60:012016, 2014.

[42] Reiner Dietsch, Stefan Braun, Thomas Holz, Hermann Mai, Roland Scholz, and Lutz Bruegemann. Multilayer x-ray optics for energies E > 8 $\mathrm{keV}$ and their application in X-ray analysis. In Carolyn A. MacDonald and Ali M. Khounsary, editors, Advances in Laboratory-based X-Ray Sources and Optics, volume 4144, pages 137 - 147. International Society for Optics and Photonics, SPIE, 2000. 
[43] W. Theiß, R. Arens-Fischer, M. Arntzen, M.G. Berger, S. Frohnhoff, S. Hilbrich, and M. Wernke. Probing Optical Transitions in Porous Silicon by Reflectance Spectroscopy in the Near Infrared, Visible and UV. MRS Proceedings, 358:435, 1994.

[44] Luca Dal Negro, Claudio J. Oton, Zeno Gaburro, Lorenzo Pavesi, Patrick Johnson, Ad Lagendijk, Roberto Righini, Marcello Colocci, and Diederik S. Wiersma. Light Transport through the Band-Edge States of Fibonacci Quasicrystals. Phys. Rev. Lett., 90:055501, 2003.

[45] G. Vincent. Optical properties of porous silicon superlattices. Appl. Phys. Lett., 64(18):2367-2369, 1994.

[46] V. Agarwal and J. A. del Río. Tailoring the photonic band gap of a porous silicon dielectric mirror. Appl. Phys. Lett., 82(10):1512-1514, 2003.

[47] D. Estrada-Wiese, Jesus Del Río, and Ehecatl del Rio-Chanona. Stochastic optimization of broadband reflecting photonic structures. Sci. Rep.-UK, 8:1193, 2018.

[48] W. Luis Mochán and Marcelo del Castillo Mussot. Optics of multilayered conducting systems: Normal modes of periodic superlattices. Phys. Rev. B, 37:6763-6771, 1988.

[49] Pérez-Huerta J. S., Ariza-Flores D., Castro-García R., Mochán W. L., Ortiz G. P., and Agarwall V. Reflectivity of 1D photonic crystals: A comparison of computational schemes with experimental results. Int. J. Mod. Phys. B, 32(11):1850136, 2018.

[50] Xifré-Pérez E., Marsal L. F., Pallarés J., and Ferré-Borrull J. Porous silicon mirrors with enlarged omnidirectional band gap. J. Appl. Phys., 97(6):064503, 2005.

[51] Yoel Fink, Joshua N. Winn, Shanhui Fan, Chiping Chen, Jurgen Michel, John D. Joannopoulos, and Edwin L. Thomas. A dielectric omnidirectional reflector. Science, 282(5394):1679-1682, 1998. 